\title{
Extrapulmonary involvement in patients with sarcoidosis and comparison of routine laboratory and clinical data to pulmonary involvement
}

\author{
Monika Zurkovaa , Vitezslav Kolek, ${ }^{\mathrm{a}}$, Tereza Tomankova ${ }^{\mathrm{b}}$, Eva Kriegova ${ }^{\mathrm{b}}$
}

\begin{abstract}
Introduction. Patients with pulmonary and pulmonary plus extrapulmonary sarcoidosis differ in symptom severity and health status impairment. To date there is no information on differences in clinical and laboratory parameters between these phenotypes and limited information on extrapulmonary involvement in Czech sarcoidosis patients exists. Methods. We therefore compared clinical data (age, gender, organ involvement, lung function tests) and laboratory data (blood counts, bronchoalveolar fluid (BAL) cellular profile, serum levels of CRP, SACE, sIL-2R, neopterin) between patients with newly diagnosed pulmonary sarcoidosis $(n=107)$ and those with pulmonary plus extrapulmonary sarcoidosis $(n=54)$.

Results. Extrapulmonary sarcoidosis was diagnosed in 33\% of patients, mostly affecting lymph nodes and skin and having hypercalciuria. There was no difference in the prevalence of extrapulmonary sarcoidosis between genders. Patients with extrapulmonary sarcoidosis were older and mostly non-smokers when compared to those with limited pulmonary form. X-ray Stage I and erythema nodosum were less frequent in extrapulmonary disease. Serum levels of CRP, SACE, sIL-2R and neopterin and BAL cellular profile did not differ between both phenotypes. We observed lower platelets, FEV1, VC, and BAL CD19+ in females with extrapulmonary involvement than in those with pulmonary disease. Conclusion. Affected lymph nodes, skin and hypercalciuria were the most common in sarcoidosis patients with extrapulmonary involvement. Pulmonary sarcoidosis did not differ in clinical and routine laboratory parameters from pulmonary plus extrapulmonary sarcoidosis. Observation of low platelets, VC, FEV1 and BAL CD19+ in females with extrapulmonary sarcoidosis needs further verification in larger cohort.
\end{abstract}

Key words: serum biomarkers, bronchoalveolar lavage, lung function, lung inflammation, sarcoidosis

Received: March 3, 2014; Accepted: May 16, 2014; Available online: June 25, 2014

http://dx.doi.org/10.5507/bp.2014.026

${ }^{a}$ Department of Respiratory Medicine, Faculty of Medicine and Dentistry, Palacky University Olomouc and University Hospital Olomouc, Czech Republic

${ }^{b}$ Department of Immunology, Faculty of Medicine and Dentistry, Palacky University Olomouc

Corresponding author: Eva Kriegova, e-mail:eva.kriegova@email.cz

\section{INTRODUCTION}

Sarcoidosis is a systemic granulomatous disease of unknown aetiology. It commonly affects young and middle aged adults, more frequently women and non-smokers ${ }^{1}$. The prevalence of sarcoidosis around the world varies between 6-213/100000 inhabitants, depending on the race and population. In the Czech Republic, a Middle European country, the incidence is 3.1/100000 and the prevalence more than $70 / 100000$ inhabitants $^{2}$.

The most common presentation of sarcoidosis is bilateral hilar lymphadenopathy and pulmonary infiltration. However, the pulmonary involvement is commonly accompanied by the involvement of other organs. The most commonly affected organs are eyes, liver, spleen, lymph nodes, salivary glands, heart, nervous system, muscles, bones, but also other organs such as uterine, stomach, bone marrow and others may be rarely affected ${ }^{1}$. The affected organs and the prevalence of extrapulmonary sarcoidosis vary between populations. A Case Control Study of Sarcoidosis (ACCESS) demonstrated that there is more extrapulmonary sarcoidosis in African-Americans than in Caucasians and different organs are affected within races ${ }^{3,4}$. Caucasians have more frequently a hypercalciuria, a disorder of calcium metabolism typical by excessive urinary calcium excretion, related to sarcoidosis, and African-Americans involvement of eyes, liver, bone marrow, peripheral lymph nodes and $\operatorname{skin}^{3}$. In Japanese patients, there are common cardiac and eye lesions ${ }^{5,6}$, in Brazilians skin, peripheral lymph system, heart and defects in calcium metabolism ${ }^{7}$ and in Turkish patients skin and peripheral lymph nodes ${ }^{8}$ are most often affected. For condition with simultaneous pulmonary and extrapulmonary involvements, the term "generalized" sarcoidosis ${ }^{3}$ has been introduced. Recently, a new term "extensive" sarcoidosis has been used for systemic involvement of more than two organs except of involving of extrathoracical lymph nodes?.

Extrapulmonary sarcoidosis may be on occasion life threatening and may also affect the therapeutic approach ${ }^{9}$. There is also evidence that patients with pulmonary plus extrapulmonary sarcoidosis are more impaired in the severity of symptoms, restrictions of activities of daily living and there is also impairment of health status comparing to the patients with isolated pulmonary sarcoidosis ${ }^{10}$. One may therefore expect that pulmonary sarcoidosis 
differ in clinical and laboratory parameters from those patients with accompanied extrapulmonary sarcoidosis. However, limited data is available on this topic.

In this study, we aimed to characterize the extrapulmonary sarcoidosis in Czech patients, because there is no information on organ involvement and frequency of extrapulmonary involvement in sarcoidosis patients from Middle Europe. We aimed also to investigate whether patients with pulmonary sarcoidosis differ in routinely assessed clinical and laboratory data from those with accompanied extrapulmonary involvement.

\section{MATERIAL AND METHODS}

\section{Subjects}

We performed a retrospective review of clinical and laboratory data in a cohort of 161 sarcoidosis patients
(91 women, the mean age 50 yrs; 70 men, $37 \mathrm{yrs)} \mathrm{di-}$ agnosed between January 2007 and December 2011 at the Department of Respiratory Medicine in Olomouc, a tertiary referral centre for interstitial lung diseases in Moravia. Subanalysis was performed in 107 patients with isolated pulmonary involvement (61 women, 48 yrs; 46 men, 36 yrs) and 54 patients with accompanying extrapulmonary involvement ( 30 women, 56 yrs; 24 men, $38 \mathrm{yrs}$ ); further subanalysis was done in subgroups according to the gender. Organ involvement was evaluated according to the ACCESS protocol ${ }^{11}$. We evaluated age, sex, smoking, X-ray stages and lung function tests (FEV1\%, FEV1, VC, VC\%, DLCO\% and TLC). Respiratory tests were done by Master Screen Body plethysmograph (Jaeger GmbH \& Co., Würzburg, Germany) at the time of diagnosis. Levels of serum biomarkers - CRP, serum angiotensin-converting enzyme (SACE), soluble interleukin-2 receptor (sIL-2R) (Roche, Mannheim, Germany) and

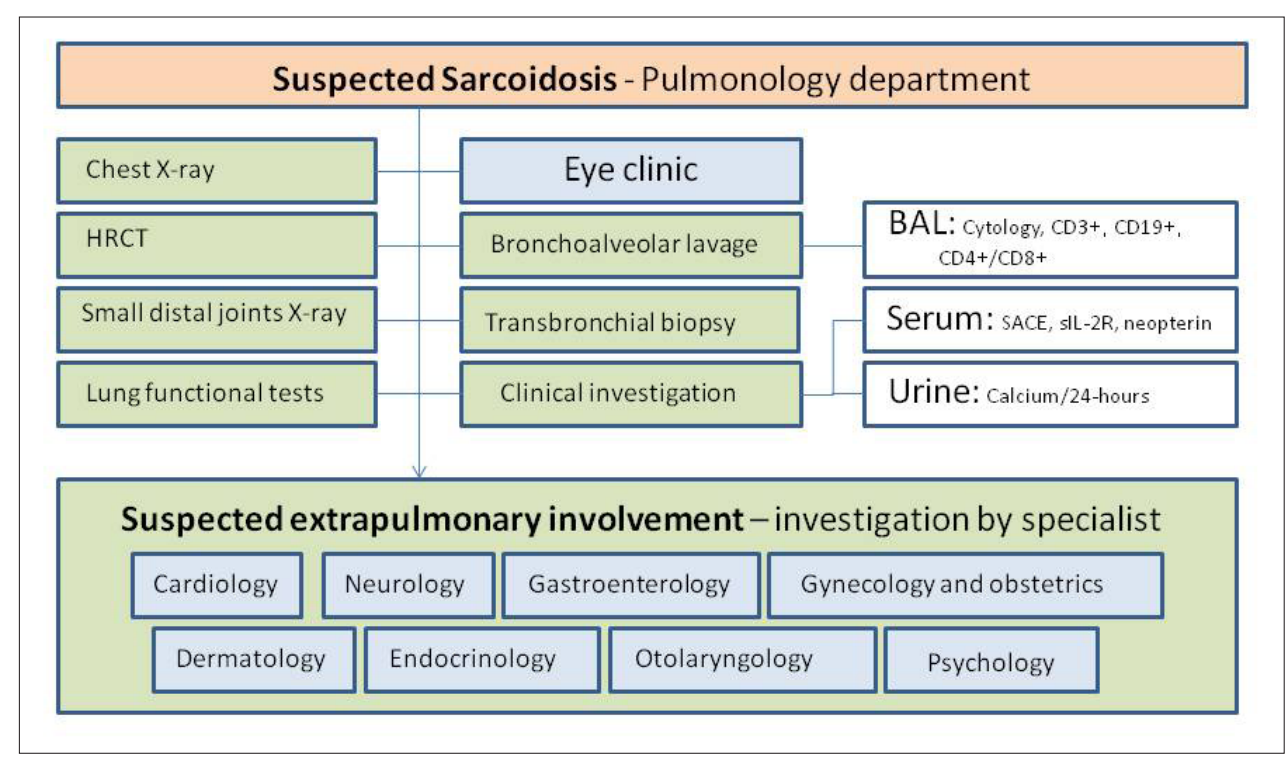

Fig. 1. Workflow of routine investigations of sarcoidosis patients.

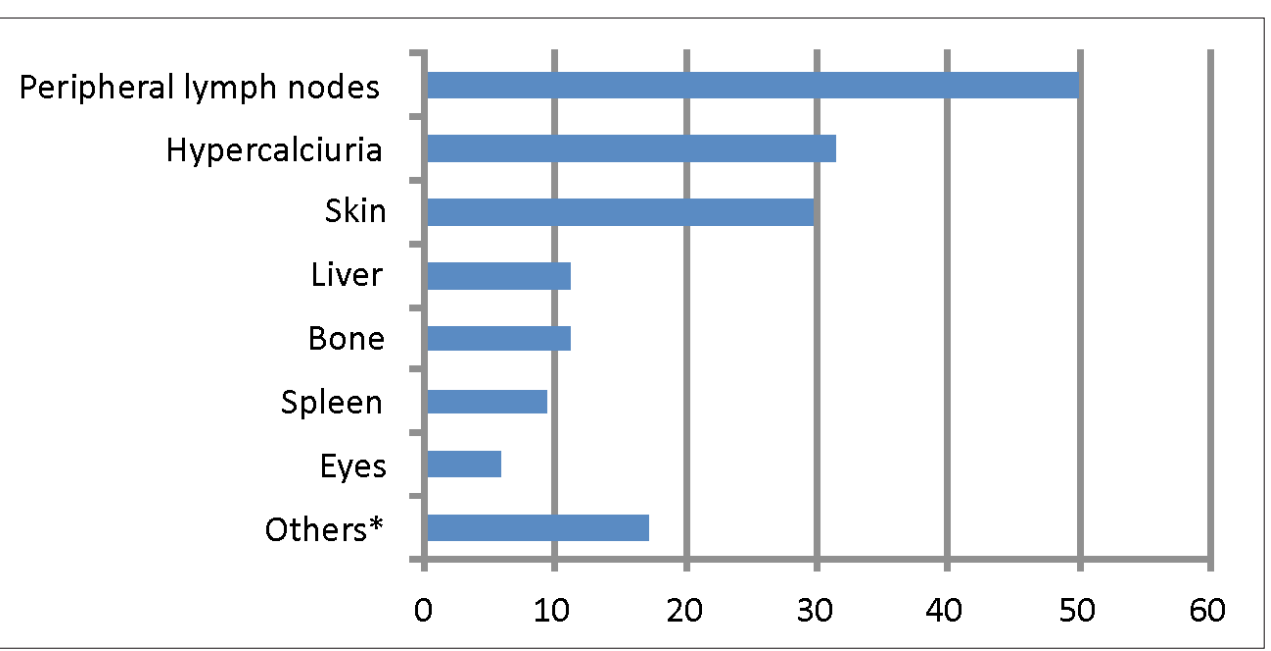

Fig. 2. Organ involvements and calcium metabolism defects (hypercalciuria) in patients with extrapulmonary sarcoidosis. On the x-axis is the number of affected patients in \%.

*Single cases of affected central nervous system, uterus, stomach, salivary glands, peritoneum, and bone marrow. 

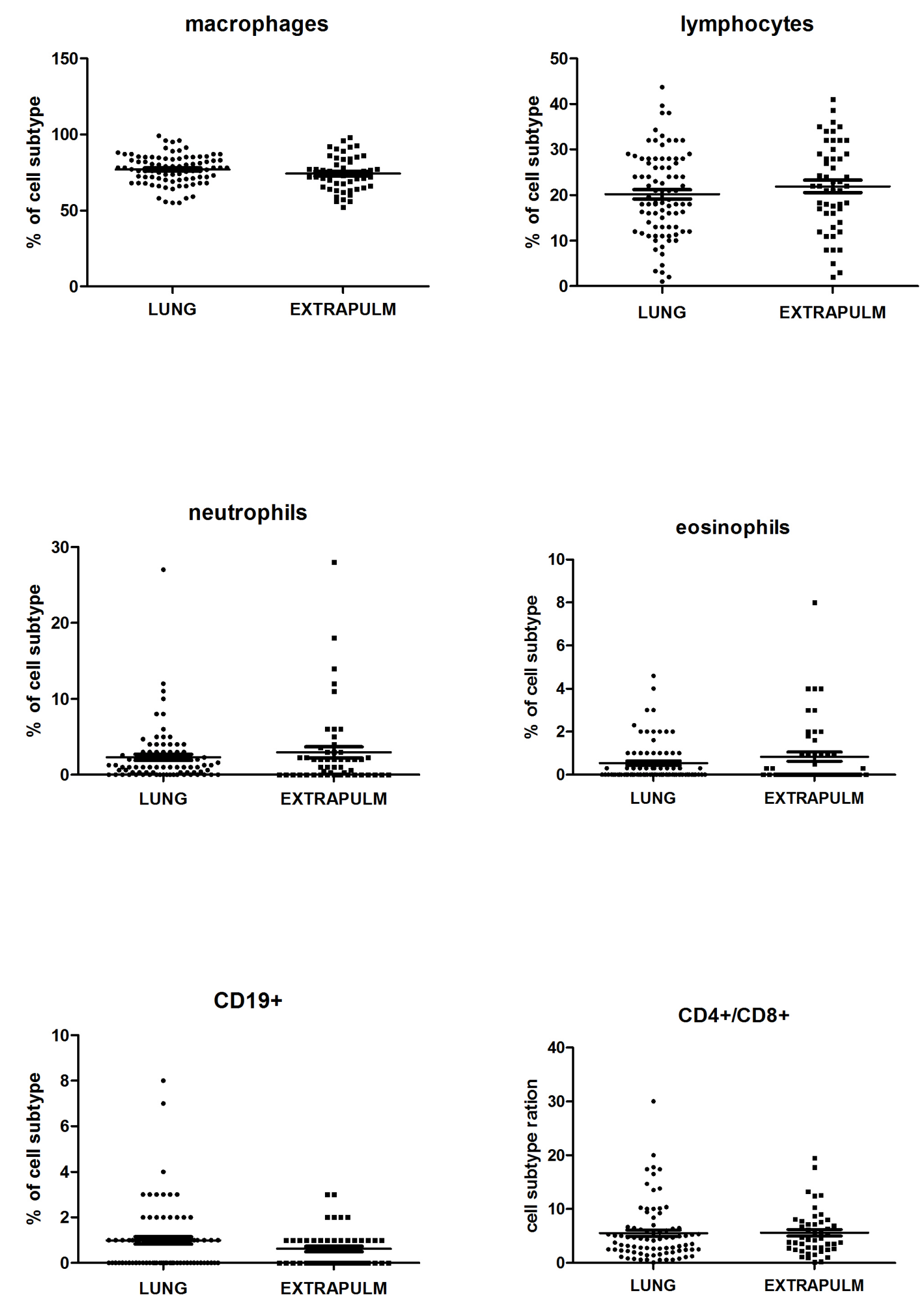

Fig. 3. Distribution of cellular subpopulation profiles and ration $\mathrm{CD} 4+/ \mathrm{CD} 8+$ in bronchoalveolar lavage fluid in patients with pulmonary (LUNG, $n=89$ ) and pulmonary plus extrapulmonary sarcoidosis (EXTRAPULM, n=51). (A) macrophages, (B) lymphocytes, (C) neutrophils, (D) eosinophils, (E) CD19+, (F) CD4+/CD8+. Group means are indicated by horizontal bars, error bars indicate SEM; $P$-values did not reach significance between pulmonary and pulmonary plus extrapulmonary sarcoidosis. 
Table 1. Patient's characteristics.

\begin{tabular}{lccc}
\hline Parameters & $\begin{array}{c}\text { Sarcoidosis }(\mathrm{S}) \\
(\mathrm{n}=161)\end{array}$ & $\begin{array}{c}\text { Pulmonary plus extrapul- } \\
\text { monary S }(\mathrm{n}=54)\end{array}$ & $\begin{array}{c}\text { Pulmonary S } \\
(\mathrm{n}=107)\end{array}$ \\
\hline Sex (men/women) & $70 / 91$ & $24 / 30$ & $46 / 61$ \\
Age & $46.0(35.0-56.0)$ & $49.0(36.8-60.3)^{*}$ & $45.0(33.0-53.0)^{*}$ \\
Females & $50.0(41.0-58.0)$ & $56.0(44.3-61.3)^{*}$ & $48.0(38.5-55.5)^{*}$ \\
Men & $37.0(32.0-47.3)$ & $38.0(35.3-56.3)$ & $36.0(29.8-46.0)$ \\
Chest X-ray stage (I/II/III/IV) & $49 / 101 / 11 / 0$ & $8 / 39 / 7 / 0$ & $41 / 62 / 4 / 0$ \\
Erythema nodosum/LS & $18 / 18$ & $1 / 1$ & $17 / 17$ \\
Smokers/ Non-smokers/ Former smokers & $30 / 98 / 33$ & $10 / 38 / 6$ & $20 / 60 / 27$ \\
\hline
\end{tabular}

All values are presented as medians, in brackets are 25.-75. percentile. LS Lögren's syndrome $* \mathrm{P}<0.05$

Table 2. Laboratory data in sarcoidosis patients subgrouped according to the involved organs.

\begin{tabular}{|c|c|c|c|}
\hline Laboratory data & $\begin{array}{c}\text { Pulmonary plus } \\
\text { extrapulmonary } \mathrm{S} \\
(\mathrm{N}=54)\end{array}$ & $\begin{array}{l}\text { Pulmonary S } \\
\qquad(\mathrm{N}=107)\end{array}$ & $P$ \\
\hline \multicolumn{4}{|l|}{ Serum biomarkers } \\
\hline $\mathrm{CRP}[\mathrm{mg} / \mathrm{L}]$ & $3.9(2.0-7.8)$ & $4.8(2.0-13.0)$ & 0.37 \\
\hline SACE [U] & $53.7(37.9-69.2)$ & $50.2(37.8-64.4)$ & 0.27 \\
\hline Neopterin $[\mu \mathrm{g} / \mathrm{L}]$ & $2.2(1.5-3.9)$ & $2.0(1.6-2.6)$ & 0.35 \\
\hline sIL-2R [kU/L] & $899(574-1338)$ & $847(414-1139)$ & 0.48 \\
\hline \multicolumn{4}{|l|}{ Blood counts } \\
\hline Leukocytes $\left[\mathrm{x} 10^{9} / \mathrm{L}\right]$ & $5.8(4.5-7.0)$ & $6.1(5.1-7.7)$ & 0.11 \\
\hline Lymphocytes $\left[\times 10^{9} / \mathrm{L}\right]$ & $1.59(1.12-1.90)$ & $1.30(1.00-1.80)$ & 0.15 \\
\hline Hemoglobin $[\mathrm{g} / \mathrm{L}]$ & $140(128-152$ & $138(128-153)$ & 0.94 \\
\hline Trombocytes $\left[\times 10^{9} / \mathrm{L}\right]$ & $241(202-291)$ & $253(212-316)$ & 0.16 \\
\hline \multicolumn{4}{|l|}{ Pulmonary functional tests } \\
\hline FEV1 [\%] & $98.5(82.0-110.5)$ & $99.0(88.0-109.0)$ & 0.79 \\
\hline FEV1 [L] & $2.9(2.2-3.6)$ & $3.1(2.6-3.7)$ & 0.22 \\
\hline $\mathrm{VC}[\%]$ & $106(84-118)$ & $105(95-119)$ & 0.62 \\
\hline $\mathrm{VC}[\mathrm{L}]$ & $3.7(3.1-4.6)$ & $4.1(3.5-4.9)$ & 0.18 \\
\hline DLCO [\%] & $92(80-102)$ & $88(80-102)$ & 0.87 \\
\hline TLC [\%] & $103(93-110)$ & $103(94-112)$ & 0.65 \\
\hline \multicolumn{4}{|c|}{ BAL profile: Relative counts [\%] ${ }^{\#}$} \\
\hline Macrophages & $74.0(65.4-83.7)$ & $78.5(70.4-84.4)$ & 0.14 \\
\hline Lymphocytes & $22.0(16.0-29.0)$ & $19.0(13.0-28.0)$ & 0.30 \\
\hline Neutrophils & $1.0(0.0-3.0)$ & $1.0(0.3-3.0)$ & 0.77 \\
\hline Eosinophils & $0.0(0.0-1.0)$ & $0.0(0.0-0.8)$ & 0.82 \\
\hline \multicolumn{4}{|c|}{ BAL profile: Absolute counts [count $\left.\times 10^{6} / \mathrm{mL}\right]^{\#}$} \\
\hline Macrophages & $51.3(37.2-102.8)$ & $65.9(40.8-91.3)$ & 0.40 \\
\hline Lymphocytes & $1638(748-2176)$ & $1397(758-2238)$ & 0.38 \\
\hline Neutrophils & $45(0-176)$ & $51(0-147)$ & 0.85 \\
\hline Eosinophils & $0(0-12)$ & $0(0-6)$ & 0.35 \\
\hline BAL CD3+ (\%) & $85(68-91)$ & $86(73-92)$ & 0.61 \\
\hline BAL CD4+ (\%) & $64(46-76)$ & $63(43-75)$ & 0.83 \\
\hline BAL CD8+ (\%) & $13(9-20)$ & $15(9-24)$ & 0.24 \\
\hline BAL CD19+ (\%) & $0(0-1)$ & $1(0-1)$ & 0.17 \\
\hline BAL CD4+/CD8+ ratio & $4.30(2.65-7.60)$ & $4.40(2.20-6.45)$ & 0.47 \\
\hline
\end{tabular}

All value are presented as medians, in brackets are 25.-75. percentiles.

\# BAL cytology was available in 51 patients with pulmonary plus extrapulmonary sarcoidosis (S) and 89 patients with limited pulmonary sarcoidosis. SACE - Serum angiotensin-converting enzyme, sIL-2R - soluble interleukin-2 receptor. 
Table 3. Comparison of laboratory and clinical data in subgroups according to the gender and sarcoidosis phenotype.

\begin{tabular}{|c|c|c|c|c|c|c|}
\hline & \multicolumn{3}{|c|}{ Male } & \multicolumn{3}{|c|}{ Female } \\
\hline & $\begin{array}{c}\text { Pulmonary } \\
\text { plus extrapulmonary } \\
S(n=24)\end{array}$ & $\begin{array}{l}\text { Pulmonary S } \\
(\mathrm{n}=46)\end{array}$ & $P$ & $\begin{array}{c}\text { Pulmonary } \\
\text { plus extrapulmonary } \\
S(n=30)\end{array}$ & $\begin{array}{l}\text { Pulmonary } S \\
\qquad(n=61)\end{array}$ & $P$ \\
\hline $\begin{array}{l}\text { Smoking status } \\
\text { Smokers/ } \\
\text { ex-smokers/ } \\
\text { non-smokers }\end{array}$ & $7 / 2 / 15$ & $13 / 15 / 18$ & 0.05 & $3 / 4 / 23$ & $7 / 12 / 42$ & 0.82 \\
\hline Age at diagnosis & $38.0(32.3-56.3)$ & $36.0(29.8-46.0)$ & 0.10 & $56.0(44.3-61.3)$ & $48.0(38.5-55.5)$ & 0.04 \\
\hline \multicolumn{7}{|l|}{ Serum biomarkers } \\
\hline $\mathbf{C R P}[\mathrm{mg} / \mathrm{L}]$ & $3.9(2.3-9.3)$ & $3.0(1.2-10.0)$ & 0.65 & $3.8(1.9-7.1)$ & $6.0(2.1-16.3)$ & 0.12 \\
\hline SACE [U] & $59.7(46.7-73.6)$ & $52.3(39.1-64.0)$ & 0.20 & $52.8(36.2-63.3)$ & $47.6(37.0-65.1)$ & 0.89 \\
\hline SACE $[<60 \mathrm{U} />60 \mathrm{U}]$ & $13 / 11$ & $33 / 13$ & 0.19 & $22 / 8$ & $44 / 17$ & 1.00 \\
\hline Neopterin $[\mu \mathrm{g} / \mathrm{L}]$ & $2.55(1.77-4.60)$ & $1.96(1.38-2.60)$ & 0.08 & $1.87(1.49-3.37)$ & $2.10(1.70-2.72)$ & 0.86 \\
\hline sIL-2R [kU/L] & $1018(777-1437)$ & $884(399-1136)$ & 0.18 & $683(394-1246)$ & $802(416-1148)$ & 0.75 \\
\hline \multicolumn{7}{|c|}{ Pulmonary functional tests } \\
\hline$\overline{\text { FEV1 [\%] }}$ & $97(78-109)$ & $94(80-105.25)$ & 0.81 & $99(87-113)$ & $101(93-113)$ & 0.62 \\
\hline FEV1 [L] & $3.55(3.35-4.43)$ & $3.64(3.08-4.25)$ & 0.94 & $2.46(2.02-2.89)$ & $2.89(2.39-3.26)$ & 0.02 \\
\hline $\mathrm{VC}[\%]$ & $96(85-111)$ & $98(87-105)$ & 0.78 & $113(97-121)$ & $113(102-124)$ & 0.56 \\
\hline $\mathrm{VC}[\mathrm{L}]$ & $4.92(4.38-5.54)$ & $4.79(4.12-5.36)$ & 0.68 & $3.22(2.80-3.65)$ & $3.73(3.32-4.14)$ & 0.004 \\
\hline DLCO [\%] & $93(81-106)$ & $90(78-104)$ & 0.78 & $91(78-100)$ & $88(80-102)$ & 0.92 \\
\hline TLC [\%] & $97(87-110)$ & $98(92-106)$ & 0.94 & $104(98-111)$ & $107(100-115)$ & 0.43 \\
\hline \multicolumn{7}{|c|}{ BAL profile: Relative counts [\%] ${ }^{\#}$} \\
\hline Macrophages & $70.5(64.5-84.8)$ & $77.0(68.0-83.3)$ & 0.27 & $75.0(69.5-81.1)$ & $78.0(70.7-85.0)$ & 0.34 \\
\hline Lymphocytes & $26.5(13.8-32.5)$ & $18.0(14.5-27.3)$ & 0.23 & $21.0(16.5-26.2)$ & $19.6(12.0-28.0)$ & 0.78 \\
\hline Neutrophils & $1.0(0-2.5)$ & $1.0(0-4.0)$ & 0.80 & $2.0(0-3.5)$ & $1.3(0.3-3.0)$ & 0.91 \\
\hline Eosinophils & $0(0-1.2)$ & $0(0-0.7)$ & 0.86 & $0(0-1.0)$ & $0.3(0-1.0)$ & 0.90 \\
\hline \multicolumn{7}{|c|}{ BAL profile: Absolute counts [count $\left.\times 10^{6} / \mathrm{mL}\right]^{\#}$} \\
\hline Macrophages & $59.5(43.5-120.2)$ & $78.6(48.9-94.4)$ & 0.66 & $46.0(34.1-86.5)$ & $58.9(36.8-80.2)$ & 0.44 \\
\hline Lymphocytes & $1596(755-2896)$ & $1400(699-2272)$ & 0.23 & $1638(698-2057)$ & $1324(783-2238)$ & 0.90 \\
\hline Neutrophils & $36(0-152)$ & $35(0-146)$ & 0.86 & $65(0-212)$ & $73(0-148)$ & 0.98 \\
\hline Eosinophils & $0(0-17.8)$ & $0(0-4.5)$ & 0.29 & $0(0-11.5)$ & $0(0-6.0)$ & 0.71 \\
\hline$\overline{\mathrm{CD} 3+(\%)}$ & $87(62-91)$ & $88(82-92)$ & 0.42 & $84(71-92)$ & $85(69-91)$ & 0.87 \\
\hline $\mathrm{CD} 4+(\%)$ & $64(38-77)$ & $63(43-74)$ & 0.98 & $65(51-77)$ & $62(41-77)$ & 0.83 \\
\hline $\mathrm{CD} 8+(\%)$ & $13(9-21)$ & $17(13-28)$ & 0.11 & $12.0(9-18)$ & $12.5(8-22)$ & 0.75 \\
\hline $\mathrm{CD} 19+(\%)$ & $1.0(0-1.5)$ & $0(0-1.0)$ & 0.66 & $0(0-1.0)$ & $1.0(0-1.3)$ & 0.02 \\
\hline CD4+/CD8+ & $3.86(2.30-6.96)$ & $3.43(1.99-5.55)$ & 0.57 & $4.66(2.73-7.84$ & $4.56(2.21-8.61)$ & 0.65 \\
\hline
\end{tabular}

All values are medians, in brackets is $25 .-75$. percentile.

\# BAL cytology was available in 51 patients with extrapulmonary sarcoidosis (S) and 89 patients with limited pulmonary sarcoidosis. SACE Serum angiotensin-converting enzyme, sIL-2R - soluble interleukin-2 receptor.

neopterin (ELISA, IBL International GmbH, Hamburg, Germany) and the blood counts were assessed in peripheral blood at the time of diagnosis. Similarly, the bronchoalveolar lavage (BAL) cellular profile was evaluated using FACSCalibur flow cytometer (BD Biosciences, CA, USA) at the time of diagnosis. All patients enrolled into this study were of Czech origin and were not relatives. The detailed demographic data are shown in Table 1. The flow chart of routine investigation of sarcoidosis patients is shown in Fig. 1. The local ethical committee of Palacky University and Faculty Hospital Olomouc approved the study.

\section{Statistical Analysis}

Fisher's exact test was used to compare categorical data, and quantitative comparisons were performed using unpaired t-test or Mann-Whitney test. The Shapiro-Wilk test was used to test the normality of data distribution. The statistical analysis was performed using GraphPad Prism 5.01 (GraphPad Software, La Jolla, CA, USA) and SPSS version 15.0 (SPSS Inc, Chicago, IL, USA). A $P$-value $<0.05$ was considered as significant.

\section{RESULTS}

\section{Comparison of clinical parameters}

In our patients with sarcoidosis, age median in female group was 50 years (range 41.0-58.0) (Table 1). The pulmonary plus extrapulmonary sarcoidosis was present in 54 patients $(33.3 \%)$ and the form limited to the lungs in 107 patients $(66.6 \%)$. Patients with accompanied extrapul- 
monary sarcoidosis were older than those with a limited form (median age 49 years vs. 45 years; $P=0.02$ ) (Table 1 ). Similarly, females with extrapulmonary sarcoidosis were older (median age 56.0 years) than those with only lung involvement (48.0 years; $P=0.04$ ) (Table 1). Also males with extrapulmonary involvement were older than those with the disease limited to the lungs (38 vs. 36 years), but the difference did not reach significance. There was no difference in the proportion of gender among patients with limited form and those with extrapulmonary involvement (males: $44 \%$ vs. $43 \%$; $P>0.05$ ). When assessing radiological stages, stage II was most frequent stage in both extrapulmonary (72.2\%) as well as in the limited form of sarcoidosis $(57.9 \%)$. Stage I was more frequently present in patients with pulmonary form than in extrapulmonary sarcoidosis (38.3\% vs $14.8 \%)$, as well as erythema nodosum (EN; $16.8 \%$ vs $1.9 \%)$. In all patients EN was associated with Löfgren's syndrome.

Patients in the present study were mostly non-smokers (60.9\%) (Table 1). In the extrapulmonary sarcoidosis subgroup, there were more non-smokers $(70.4 \%$ vs. $56.1 \%)$ and fewer ex-smokers (11.1\% vs. $25.2 \%)$ than in the subgroup with the limited form, but the difference did not reach significance $(P=0.09)$ (Table 1$)$.

\section{Affected organs and calcium metabolism defects associ- ated with sarcoidosis}

In patients with extrapulmonary involvement, the most commonly affected organs were peripheral lymph nodes (50.3\%), skin (29.6\%), liver (11.1\%), and spleen $(9.3 \%)$. The eyes $(5.7 \%)$ and the uterus, stomach, lacrimal glands, peritoneum, and bone marrow (all 1.9\%) were involved rarely (Fig. 2). About one-third of patients (31.5\%) had a hypercalciuria. In our cohort, we found lower prevalence of hypercalciuria (7.7\% vs $14.3 \%)$ and peripheral lymph node involvement ( $13.2 \%$ vs $21.4 \%$ ) in women than in men, but the difference did not reach significance.

Regarding number of diseased organs, the majority of patients with extrapulmonary sarcoidosis had one additional organ except lungs affected $(75.9 \%)$, followed by two extrapulmonary organs affected $(13.0 \%)$ and three and more organs affected $(13.0 \%)$. In subgroups according to the gender, we found more women with one extrapulmonary affected organ (83.3\% vs $66.7 \%)$ and fewer women with three and more affected organs $(6.7 \%$ vs $20.8 \%$ ) than in men, but the difference did not reach significance.

\section{Comparison of laboratory and functional parameters between patients with different clinical phenotypes}

No difference in hemoglobin, leukocyte, lymphocyte and platelet counts was observed between the groups with pulmonary and extrapulmonary sarcoidosis at the time of diagnosis $(P>0.05)$. Both groups did not differ in serum concentrations of CRP, SACE, sIL-2R and neopterin ( $P>0.05$, Table 2$)$. Moreover, there were no marked obvious differences in functional parameters: FEV1 [\%], FEV1, VC [\%], VC, DLCO [\%], TLC [\%] $(P>0.05)$ between patients with both studied phenotypes (Table 2 ).
Higher platelet count ( 268 vs. $24410^{9} / \mathrm{L} ; P=0.04$ ), higher VC ( 3.73 vs. $3.22 \mathrm{~L} ; P=0.004)$ and higher FEV1 ( 2.89 vs. $2.46 \mathrm{~L} ; P=0.02$ ) was observed in females with pulmonary sarcoidosis than in those with the extrapulmonary form (Table 3). These differences were not found in male subgroup.

\section{Comparison on the BAL fluid cellular profile between patients with different clinical phenotypes}

Data on the BAL cellular profile and CD4+/CD8+ ratio and other immunophenotyping results were available in 140 patients with sarcoidosis enrolled in the study. No differences were found in either absolute or relative counts of alveolar macrophages, lymphocytes, neutrophils and eosinophils between patients with limited and generalized forms ( $P>0.05$, Table 2, Fig. 3) as well as in gender subgroups ( $P>0.05$, Table 3$)$. In female patients, there was higher proportion of CD19+ lymphocytes in the BAL fluid obtained from patients with pulmonary sarcoidosis than in those with the extrapulmonary form of the disease $(P=0.02)$ (Table 3$)$.

\section{DISCUSSION}

In the years 2007-2011, a total of 161 new cases of sarcoidosis were diagnosed at a tertiary centre for interstitial lung diseases in Moravia. Of those, $67 \%$ of patients were diagnosed with pulmonary sarcoidosis and 33\% had in addition extrapulmonary involvement. In extrapulmonary group, the most frequently affected organs were peripheral lymph nodes and skin, and one-third of patients had a hypercalciuria. Although the patients with pulmonary and pulmonary plus extrapulmonary sarcoidosis differ in symptom severity and health status impairment ${ }^{10}$, we did not observe any significant difference in clinical and laboratory parameters between these phenotypes. Interestingly, we observed higher levels of platelet count, functional parameters VC and FEV1 and CD19+ lymphocytes in the bronchoalveolar fluid in females with pulomonary sarcoidosis than in those with accompanying extrapulmonary disease.

Our patients with pulmonary plus extrapulmonary disease were older than those with only pulmonary sarcoidosis; the same trend was observed in subgroups according to the gender. Although higher frequency of extrapulmonary sarcoidosis has been reported in females than in males ${ }^{3}$, in our study cohort the percentages of males and females with extrapulmonary sarcoidosis were identical. However, women had tendency to less generalized disease as assessed by number of affected organs. It is also of interest that there were more non-smokers among patients with extrapulmonary sarcoidosis than among those with the limited pulmonary form, particularly in the male subgroup.

In our patients with extrapulmonary involvement, the most commonly affected organs were peripheral lymph nodes. Lymphadenomegaly may develop at any site, the cervical, axillary, epitrochlear and inguinal nodes become 
enlarged most frequently. The frequency of peritoneal and retroperitoneal nodes is unknown, because they tend to be clinically silent ${ }^{12,13}$. Node enlargement was mostly mild, with the nodes being hard but not tender or ulcerating. Another common manifestation associated with sarcoidosis in our patients was a hypercalciuria $(31.5 \%)$, a frequent form of calcium metabolism abnormality in Caucasians $^{14}$. Skin involvement, seen in about $25 \%$ of patients worldwide ${ }^{14}$, was confirmed in about $30 \%$ of our extrapulmonary sarcoidosis cases. Interestingly, eye involvement reported in 10-50\% of European cases and $50-90 \%$ of Japanese cases ${ }^{14}$, was infrequent in our patients $(5.7 \%)$. Besides involvement of liver and spleen, we observed also rarely affected organs such as uterus, stomach, lacrimal glands, and peritoneum. Importantly, there is a discrepancy in the literature regarding the categorizing of erythema nodosum (EN). Some authors include EN into the extrapulmonary involvement ${ }^{7,11}$, however we and others ${ }^{15}$ believe that $\mathrm{EN}$ is just reaction not the extrapulmonary form of sarcoidosis. Moreover, EN is associated with a good prognosis, often resolving spontaneously, which is in contrast to extrapulmonary sarcoidosis. Therefore, we did not include EN into the extrapulmonary form of disease.

Although $30-50 \%$ of patients with pulmonary sarcoidosis are known to have extrapulmonary involvement often requiring different treatment options, little is known about differences in clinical and laboratory parameters between patients with the disease limited to the lungs and those with the extrapulmonary form. In contrast to our expectation, we did not observe any differences in functional lung parameters as well as in blood count values between both phenotypes. When subdivided according to the gender, females with extrapulmonary disease had lower platelet count and lower VC and FEV1 than those with the limited pulmonary form of sarcoidosis. This may be explained by the fact that our females with the extrapulmonary form were older than their counterparts with limited disease and/or it may relate to the affected organs and severity of disease in female subgroup. It can be also assumed that the lung involvement can be more intensive in limited pulmonary form. Regarding platelet count, low number of platelet counts has been already reported in patients with affected spleen ${ }^{16-19}$ and German patients with sarcoidosis $^{20}$. The association of platelet counts and severity of disease, resp. affected organs should be further investigated. Regarding affected organs in women, ACCESS found a higher prevalence of eye ( $13.9 \%$ vs $8.2 \%)$, and neurological sarcoidosis (6.0\% vs $2.2 \%)$ and lower prevalence of hypercalciuria $(2.1 \%$ vs $6.3 \%)$ in females than in males ${ }^{4}$. Also in our patient cohort, other organs and their combination were affected by the disease in women and men. We found lower prevalence of hypercalciuria $(7.7 \%$ vs $14.3 \%)$ and patients with affected peripheral lymph nodes $(13.2 \%$ vs $21.4 \%)$ in women than in men, but the difference did not reach significance due to low number of cases.

It has been proposed that the disease activity biomarkers such as CRP, SACE, sIL-2R and neopterin can in- form about the total mass of granulomas. In our study, the serum levels of these markers did not differ between extrapulmonary and pulmonary sarcoidosis. This observation let us speculate that the number of organs affected by sarcoidosis does not correspond to amount of granulomas in the body and/or latent involvement of other organs may also play a role. In another study, out of 92 pro-inflammatory proteins higher levels of only seven analytes-CRP, CA125, CD40, ferritin, TNF RII, IL-18 and $\beta-2$ microglobulin were found in patients on anti-tumor necrosis factor therapy suffering from extrapulmonary sarcoidosis comparing to those with limited pulmonary form ${ }^{21}$.

Regarding the BAL fluid, we hypothesized that disease limited to the lung may be associated with higher numbers of inflammatory cells than BAL from patients with accompanied extrapulmonary involvement due to the possible redistribution of immune cells into other involved organs. However, we found no differences in the BAL fluid cellular profile in either absolute or relative counts of alveolar macrophages, lymphocytes, neutrophils and eosinophils between the limited pulmonary and extrapulmonary disease groups. There were also no differences in the proportion of CD4+ and CD8+ T-lymphocytes and in the CD4+/CD8+ ratio in studied subgroups. According to our knowledge, this is the first study comparing the BAL fluid profile in respect to organ involvement. Previous studies on the predictive values of BAL found that the lymphocyte count, CD4+ and CD4+/CD8+ are not indicators of prognosis on which therapeutic decisions can be based ${ }^{22}$.

Next, we aimed to investigate whether studied parameters differ in subgroups according to the gender. Females with extrapulmonary involvement had significantly lower percentages of CD19+ than those with only pulmonary form. This finding is consistent with a recent study detecting CD4+, CD8+ and CD19+ lymphopenia in the peripheral blood of patients with sarcoidosis, the condition being associated with severe disease and extrapulmonary involvement ${ }^{23}$. Lower number of circulating memory B-cells has been also found in peripheral blood obtained from patients with chronic sarcoidosis comparing to inactive form and healthy subjects ${ }^{24}$. We and others ${ }^{24,25}$ speculate that in sarcoidosis, CD19+ lymphopenia may be due to suppression of lymphomagenesis, mainly through cytokine action or apoptotic pathway activation, redistribution of B-lymphocytes into the site of granuloma and/ or changes in the B-cell homeostasis. The role of B-cells in sarcoidosis should be investigated in future studies.

We are aware that this study has limitations. The major limitations are the retrospective data analysis, the small number of patients in subgroups with particular phenotype, and the fact that this is a single centre study. However, when any of the clinical or laboratory parameters would be clinically useful as a marker of extrapulmonary sarcoidosis, it would be nominated also from our study.

In conclusion, extrapulmonary sarcoidosis was diagnosed in $33 \%$ of sarcoidosis patients, mostly affecting lymph nodes and skin, and having hypercalciuria. The 
comparison of routinely assessed clinical and laboratory parameters did not reveal any differences between pulmonary and pulmonary plus extrapulmonary disease in the whole group and in male subgroup. In females with extrapulmonary sarcoidosis, we observed lower platelet count, lower FEV1 and VC and BAL CD19+ than in females with the form limited to the lung. This observation needs further verification in larger cohort.

\section{ACKNOWLEDGEMENTS}

We thank Dr. Jana Zapletalova for her kind help with statistical analysis of clinical and laboratory data. The authors declare no conflicting financial interests. This study was supported by the Czech Ministry of Health IGA MZ ČR NT/11117 and in part by LF_UP_2014_020 and TransMedChem project CZ.1.07/2.4.00/17.0015 (TT).

Authorship contributions: MZ, TT, EK: data collection and analysis; VK, EK: study design; MZ, VK, EK: manuscript writing.

Conflict of interest statement: The authors state that there are no conflicts of interest regarding the publication of this article.

\section{REFERENCES}

1. American Thoracic Society, European Respiratory Society and World Association of Sarcoidosis and Other Granulomatous Disorders. Joint Statement on sarcoidosis. Am J Respir Crit Care Med 1999;160:73655.

2. Kolek V. Epidemiological study on sarcoidosis in Moravia and Silesia. Sarcoidosis 1994;11:110-2.

3. Baughman RP, Teirstein AS, Judson MA, Rossman MD, Yeager $H$ Jr, Bresnitz EA, DePalo L, Hunninghake G, lannuzzi MC, Johns CJ McLennan G, Moller DR, Newman LS, Rabin DL, Rose C, Rybicki B, Weinberger SE, Terrin ML, Knatterud GL, Cherniak R. Case Control Etiologic Study of Sarcoidosis (ACCESS) research group. Clinical characteristics of patients in a case control study of sarcoidosis. Am J Respir Crit Care Med 2001;164:1885-9.

4. ACCESS Research Group. Design of A Case Control Etiology Study of Sarcoidosis (ACCESS). J Clin Epidemiol 1999;52:1173-86.

5. Rybicki BA, Major M, Popovich J Jr, Maliarik MJ, lannuzzi MC. Racial differences in sarcoidosis incidence: a 5-year study in a health maintenance organisation. Am J Epidemiol 1997;145:234-41.

6. Schulte W, Kirstien D, Drent M, Costabel U. Cardiac involvement in sarcoidosis. European Respiratory Journal Monograph 2005;10:13049.

7. Rodrigues SC, Rocha NA, Lima MS, Arakaki JS, Coletta EN, Ferreira RG, Gonzaga LR, Pereira CA. Factor analysis of sarcoidosis phenotypes at two referral centers in Brazil. Sarcoidosis Vasc Diffuse Lung Dis 2011;28:34-43.
8. Okumus G, Musellim B, Cetinkaya E, Turker H, Uzaslan E, Yenturk E, Uzun O, Saglam L, Kumbasar OO, Celik G, Annakkaya AN, Altiay G, Tabak L, Sakar A, Kiter G, Erturan S, Turktas H, Yalniz E, Akkoclu A, Ogus C, Dogan OT, Ozkan M, Aktogu S, Uzel I, Ongen G. Extrapulmonary involvement in patients with sarcoidosis in Turkey. Respirology 2011;16:446-50.

9. Rao DA, Dellaripa PF. Extrapulmonary manifestations of sarcoidosis. Rheum Dis Clin North Am 2013;39:277-97.

10. Gvozdenovic BS, Mihailovic-Vucinic V, Ilic-Dudvarski A, Zugic V, Judson MA. Differences in symptom severity and health status impairment between patients with pulmonary and pulmonary plus extrapulmonary sarcoidosis. Respir Med 2008;102:1636-42.

11. Judson MA, Baughman RP, Teirstein AS, Terrin ML, Yeager H Jr. Defining organ involvement in sarcoidosis: the ACCESS proposed instrument. ACCESS Research group. A Case Control Etiologic Study of Sarcoidosis. Sarcoidosis Vasc Diffuse Lung Dis 1999;16:75-6.

12. Semenzato G, Pezzutto A, Chilosi M, Pizzolo G. Redistribution of $T$ lymphocytes in the lymph nodes of patients with sarcoidosis. N Engl J Med 1982;306:48-9.

13. Konishi K, Moller DR, Saltini C, Kirby M, Crystal RG. Spontaneous expression of the interleukin 2 receptor gene and presence of functional interleukin 2 receptors on T lymphocytes in the blood of individuals with active pulmonary sarcoidosis. J Clin Invest 1988;82:775-81.

14. Judson MA. Extrapulmonary sarcoidosis. Semin Respir Crit Care Med 2007;28:83-101.

15. O Neill JH. The differential diagnosis of erythema nodosum. Del Med J 1991;63:683-9.

16. Wendel $\mathrm{H}$. Relation between spleen size and thrombocyte number in sarcoidosis. Z Erkr Atmungsorgane Folia Bronchol 1973;138:273-8.

17. Judson MA. Hepatic and splenic sarcoidosis. In: Baughman RP, ed. Sarcoidosis: Lung Biology in Health and Disease. New York: Marcel Dekker 2006;210:571-92.

18. Haran MZ, Feldberg E, Miller G, Berrebi A. Sarcoidosis presenting as massive splenomegaly and bicytopenia. Am J Hematol 2000;63:2323.

19. Kataria YP, Whitcomb ME. Splenomegaly in sarcoidosis. Arch Intern Med 1980;140:35-7.

20. Thadani U, Aber CP, Taylor JJ. Massive splenomegaly, pancytopenia and haemolytic anemia in sarcoidosis. Acta Haematol 1975;53:23040.

21. Loza MJ, Brodmerkel C, Du Bois RM, Judson MA, Costabel U, Drent M, Kavuru M, Flavin S, Lo KH, Barnathan ES, Baughman RP. Inflammatory profile and response to anti-tumor necrosis factor therapy in patients with chronic pulmonary sarcoidosis. Clin Vaccine Immunol 2011;18:931-9.

22. Verstraeten $A$, Demedts $M$, Verwilghen J, van den Eeckhout $A$, Mariën G, Lacquet LM, Ceuppens JL. Predictive value of bronchoalveolar lavage in pulmonary sarcoidosis. Chest 1990;98:560-7.

23. Sweiss NJ, Salloum R, Gandhi S, Alegre ML, Sawaqed R, Badaracco M, Pursell K, Pitrak D, Baughman RP, Moller DR, Garcia JG, Niewold TB. Significant CD4, CD8, and CD19 lymphopenia in peripheral blood of sarcoidosis patients correlates with severe disease manifestations. PLoS One 2010;5:e9088.

24. Saussine A, Tazi A, Feuillet S, Rybojad M, Juillard C, Bergeron A, Dessirier V, Bouhidel F, Janin A, Bensussan A, Bagot M, Bouaziz JD. Active chronic sarcoidosis is characterized by increased transitional blood B cells, increased IL-10-producing regulatory B cells and high BAFF levels. PLoS One 2012;7:e43588.

25. Selroos O, Koivunen E. Prognostic significance of lymphopenia in sarcoidosis. Acta Med Scand 1979;206:259-62. 